\title{
COMPUTED TOMOGRAPHY COMMISSIONING PROGRAMMES: HOW TO OBTAIN A RELIABLE MTF WITH AN AUTOMATIC APPROACH?
}

\author{
F. Miéville ${ }^{1, *}$, S. Beaumont ${ }^{2}$, T. Torfeh ${ }^{2}$, F. Gudinchet ${ }^{3}$ and F. R. Verdun ${ }^{1}$ \\ ${ }^{1}$ Institute of Radiation Physics (IRA), University Hospital Center and University of Lausanne, Grand Pré 1, \\ 1007 Lausanne, Switzerland \\ ${ }^{2}$ QualiFormeD SARL-Department Hospital Center, Les Oudairies, F-85925 La Roche-sur-Yon, France \\ ${ }^{3}$ Radiology Department-CHUV and UNIL, CH-1011 Lausanne, Switzerland \\ *Corresponding author: francis.verdun@chuv.ch
}

\begin{abstract}
The purpose of this study was to assess the spatial resolution of a computed tomography (CT) scanner with an automatic approach developed for routine quality controls when varying CT parameters. The methods available to assess the modulation transfer functions (MTF) with the automatic approach were Droege's and the bead point source (BPS) methods. These MTFs were compared with presampled ones obtained using Boone's method. The results show that Droege's method is not accurate in the low-frequency range, whereas the BPS method is highly sensitive to image noise. While both methods are well adapted to routine stability controls, it was shown that they are not able to provide absolute measurements. On the other hand, Boone's method, which is robust with respect to aliasing, more resilient to noise and provides absolute measurements, satisfies the commissioning requirements perfectly. Thus, Boone's method combined with a modified Catphan 600 phantom could be a good solution to assess CT spatial resolution in the different CT planes.
\end{abstract}

\section{INTRODUCTION}

Since the early 1970 s, the use of computed tomography $(\mathrm{CT})$ in the clinical environment has been steadily growing because of its proven method of diagnostic imaging. At the end of the 1990s, already more than 73000 examinations per year were performed in Switzerland and in 2003, this number was over $120000^{(1)}$. At the same time, in order to avoid a reversal of the risk-benefit ratio associated with this imaging modality, radiation dose-reduction strategies have been evolving.

Today, to ensure the accuracy of diagnostics, associations $^{(2)}$ such as the American College of Radiology (ACR, http://www.acr.org/accreditation. aspx) require that each $\mathrm{CT}$ undergo an acceptance and a commissioning programme before its first use on a patient. In addition, routine quality controls (QCs) should be performed on a regular basis to ensure stability throughout the lifetime of the CT scanner $^{(2)}$. A similar strategy is also used in Europe where acceptance and commissioning tests must also be performed.

Moreover, the acquisition protocols should comply with dose reference levels ${ }^{(3)}$. In the context of QC, automatic approaches are becoming more and more popular for performing image stability controls. The lack of a standardized method to measure image quality metrics such as the modulation transfer function (MTF), the noise power spectrum or the detector equivalent quanta has made acceptance and commissioning programmes more difficult to automate.

In this paper, MTF curves were computed with three different methods: Droege's ${ }^{(4)}$, the bead point source (BPS) and Boone's ${ }^{(5)}$ will be assessed and compared with varying $\mathrm{CT}$ acquisition parameters. The results and the methods used will be discussed in a $\mathrm{QC}$ as well as in a commissioning programme context. Finally, requirements for adapting Boone's method to an automated in-depth control of the CT spatial resolution will be proposed for commissioning programmes.

\section{MATERIALS AND METHODS}

A module composed of a $125 \mu \mathrm{m}$ aluminium foil sandwiched between two 2-cm-thick $20 \times 15 \mathrm{~cm}$ polymethylmethacrylate slabs according to Boone ${ }^{(5)}$ and a Catphan 600 phantom (Phantom Laboratory, Cambridge, NY, USA) were scanned with a 64-slice CT scanner (LightSpeed VCT, GE Healthcare, Milwaukee, WI, USA). To perform a consistent analysis, the BPS present in the Caphan 600 phantom and the aluminium foil of the module were placed at the same position relatively to the CT gantry centre.

The acquisitions were performed in the sequential mode with a fixed reconstruction slice thickness and a fixed tube voltage of $1.25 \mathrm{~mm}$ and $120 \mathrm{kVp}$, respectively. The tube current and rotation time 


\section{F. MIÉVILLE ET AL.}

values were adapted to reach $\mathrm{CTDI}_{\mathrm{vol}}$ values from 100 to $0.5 \mathrm{mGy}$. Then, the reconstructions, using the standard algorithm provided by the manufacturer, were made using display field of views (DFOVs) varying from 150 to $500 \mathrm{~mm}$ using bone, standard and soft reconstruction kernels as well as the small, medium and body scan field of views (SFOVs). The display matrix size of the CT was composed of $512 \times 512$ pixels.

The CT data files (DICOM files) were transferred from the CT unit to a standard desktop workstation for image analysis. For Droege's and the BSP methods, MTF measurements were performed using the QualiMagiQ software (QualiFormeD SARL, France), whereas an in-house program was written in MATLAB 7.7 (Mathworks, Natick, MA, USA) to implement Boone's method.

\section{Boone's method: "Reference" method}

To overcome the aliasing effect caused by the finite sampling in CT imaging, oversampling methods are usually employed. Boone's method typically allows for measuring an oversampling line spread function (LSF) from which a pre-sampled MTF is computed. This technique, considered the reference MTF throughout this work, has already shown numerous advantages ${ }^{(5)}$.

Figure 1 summarizes the different steps of this method. First, the module is slightly tilted at a $\theta$ angle and scanned. Then, a region of interest

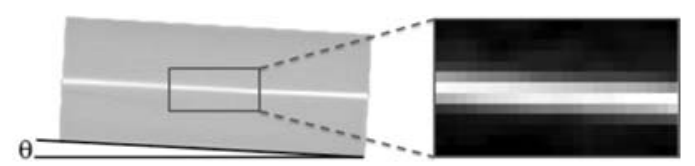

(a)
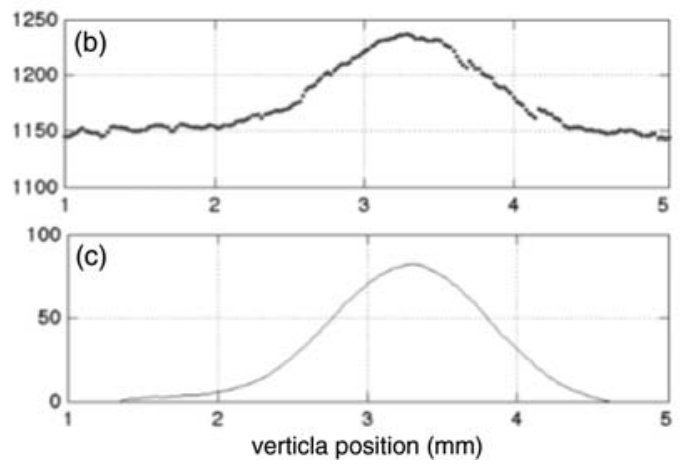

Figure 1. (a) A $125 \mu \mathrm{m}$ aluminium foil sandwiched between flat $20 \mathrm{~mm}$ plastic scanned at a $\mathrm{CTDI}_{\mathrm{vol}}=100 \mathrm{mGy}$. (b) Oversampled LSF according to Boone's method ${ }^{(6)}$.

(c) LSF using a moving average (with 15 patients on a total of 285 patients) after subtracting the background.
(ROI) of the aluminium foil is selected (a). In our program, the $\theta$ angle was determined using the angle tool of Image J 1.41 software (National Institute of Health, Bethesda, MD, USA). As described by many authors ${ }^{(6,7)}$, the theory of angling the foil produces an oversampled LSF whose oversampling rate can be increased when the $\theta$ angle is reduced relative to the horizontal (b).

In this work, the $\theta$ angle was measured as $2.5 \pm$ $0.1^{\circ}$. Then, the background value was subtracted and a slightly moving average on $p$ points (typically $p=5 \%$ of the LSF length) was used to smooth the LSF (c). The point $p$ was chosen to keep the full width at half maximum (FWHM) of the raw LSF constant. Finally, the MTF was obtained by calculating the one-dimensional Fourier transform (FT) of the smoothed LSF. Since the thickness of the aluminium foil was smaller than the smallest pixel size (for $\mathrm{DFOV}=150 \mathrm{~mm}$, pixel size $=150 / 512=$ $0.292 \mathrm{~mm}$ ), there was no need to correct by dividing the MTF with the FT of an 'ideal' foil.

\section{BPS method: the conventional method}

In the well-known BPS method, the MTF is computed from the mean of the vertical and horizontal LSFs extracted from the bead image of the CTP528 Catphan 600 phantom module. In QualiMagiQ software, the MTF is fit by an analytical Gaussian or sigmoid function ${ }^{(8)}$ using the least squares method.

Figure 2a shows the MTF with the standard reconstruction filter, a DFOV $=200 \mathrm{~mm}$ and a $\mathrm{CTDI}_{\mathrm{vol}}=100 \mathrm{mGy}$. Based on the MTF points, a sigmoid function was chosen to measure the spatial frequencies at $50 \%, 10 \%, 5 \%$ and $2 \%$ of the MTF.

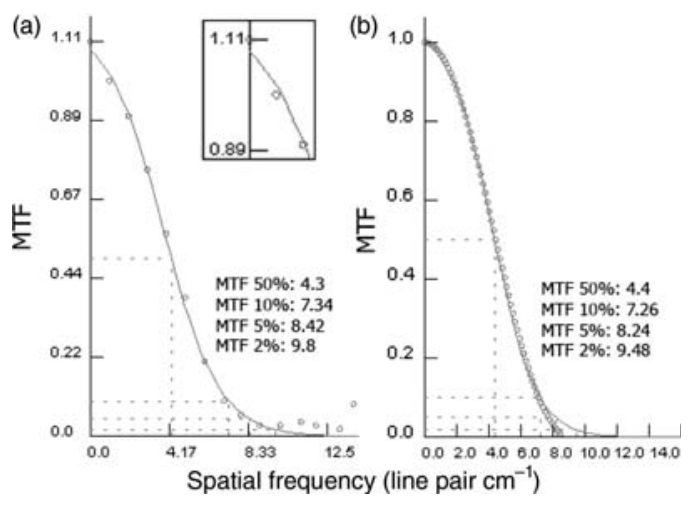

Figure 2. MTFs from the QualiMagiQ software computed using (a) the BPS method and (b) Droege's method. The acquisition was performed with a $\mathrm{DFOV}=300 \mathrm{~mm}$, the standard reconstruction filter and a $\mathrm{CTDI}_{\mathrm{vol}}=100 \mathrm{mGy}$. 


\section{Droege's method: a fast and practical method}

QualiFormeD also implemented a simple and useful method to determine the MTF of a CT image. This approach, first proposed by Droege et al. ${ }^{(6)}$, relies on the measurement of the standard deviation of the pixel values within the image of bar patterns. It was shown that for spatial frequencies $f \geq f_{\mathrm{c}} / 3$, where $f_{\mathrm{c}}$ is the cut-off frequency, the MTF could be computed using:

$$
\operatorname{MTF}(f)=\frac{\pi}{\sqrt{2}} \cdot \frac{\sqrt{\sigma^{2}(f)-\sigma_{\text {noise }}^{2}}}{\left|\mathrm{CT}_{\text {line }}-\mathrm{CT}_{\mathrm{bg}}\right|} \quad f_{\mathrm{c}} \geq \frac{f_{\mathrm{c}}}{3}
$$

where $\sigma^{2}(f)$ and $\sigma_{\text {noise }}^{2}$ are the variances measured within the bar pattern of spatial frequency $f$ and within a 'uniform' $\mathrm{ROI}$, respectively. $\mathrm{CT}_{\text {line }}$ and $\mathrm{CT}_{\mathrm{bg}}$ are the mean values of the pixels of the pattern line and the background.

This method has the advantage of being computationally faster than the conventional method based on one-dimensional or two-dimensional FT. However, for frequencies less than $f_{\mathrm{c}} / 3$, this approach diverges to reach a maximal error at zero frequency as shown in Figure $2 b$ (the high-resolution module (CTP528) of the Catphan 600 phantom was employed for these measurements).

\section{RESULTS}

The $\mathrm{CTDI}_{\mathrm{vol}}$, the reconstruction kernels and the DFOVs were varied, whereas the SFOV used throughout this work was maintained at the medium body SFOV. This choice was made because no significant variations were observed when modifying the SFOVs.

Figure 3 compares the behaviour of the presampled MTF obtained with the reference method when the DFOV varies. The increase in the pixel size when increasing the DFOV strongly affects the image characteristics. As observed, the spatial frequency at $50 \%$ of the MTF decreases from $0.337 \mathrm{~mm}^{-1}$ to $0.267 \mathrm{~mm}^{-1}$ (21\% variation) when the DFOV increases from 150 to $500 \mathrm{~mm}$. To illustrate the difference between the reference method and a conventional method, we chose a unique pixel column in Figure 1a to obtain a raw (not presampled) LSF. For a DFOV of $500 \mathrm{~mm}$, this resulted in an MTF that presented an aliasing effect owing to the low $f_{\text {nyquist }}=0.512 \mathrm{~mm}^{-1}$ (dashed curve in Figure 3).

To assess the performance of QualiMagiQ software, the spatial frequency at $50 \%$ and $5 \%$ of the MTFs were measured when varying the CT parameters and then compared with the ones obtained with the reference method. Figure 4 presents the results of the Droege, BPS and the reference

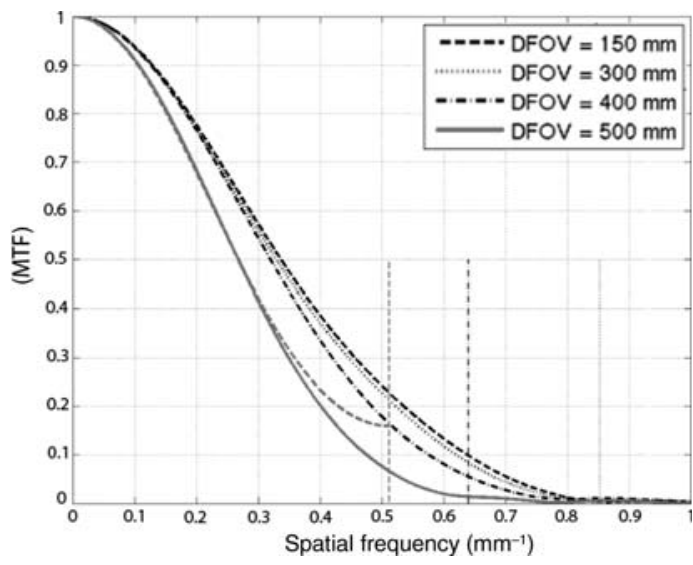

Figure 3. Behaviour of the reference (pre-sampled) MTFs when varying the DFOV for a standard reconstruction filter and a $\mathrm{CTDI}_{\mathrm{vol}}=100 \mathrm{mGy}$. The vertical lines represent the Nyquist frequencies associated with the DFOVs. For DFOV $=500 \mathrm{~mm}$, MTFs computed with the reference and the conventional methods are reported (dashed curve).

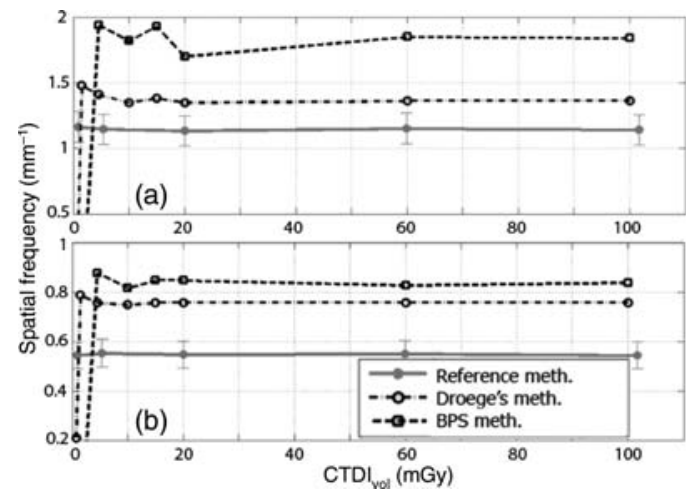

Figure 4. Comparison of spatial frequency results extracted at (a) $5 \%$ and (b) $50 \%$ of the MTFs obtained with the different methods when varying the $\mathrm{CTDI}_{\mathrm{vol}}$. The acquisitions were performed with the bone reconstruction filter and $\mathrm{DFOV}=200 \mathrm{~mm}$.

methods, when the $\mathrm{CTDI}_{\mathrm{vol}}$ varies. A DFOV of $200 \mathrm{~mm}$ was chosen to have a $f_{\text {nyquist }}$ far beyond the cut-off frequency of the MTFs. While reducing, the $\mathrm{CTDI}_{\mathrm{vol}}$ had little influence on Droege's method, even for very low $\mathrm{CTDI}_{\mathrm{vol}}$, the BSP method was more sensitive to noise. Indeed, below $\mathrm{CTDI}_{\mathrm{vol}}=$ 20-30 mGy, the uncertainty was increased. The maximum variations were less than $6.5 \%$ for spatial frequencies extracted at $5 \%$ and $50 \%$ of the MTF. Beyond a CTDI ${ }_{\mathrm{vol}}$ value smaller than $5 \mathrm{mGy}$, the automatic approach was not able to perform the measurement correctly because of the difficulty in detecting the bead. 


\section{F. MIÉVILLE ET AL.}

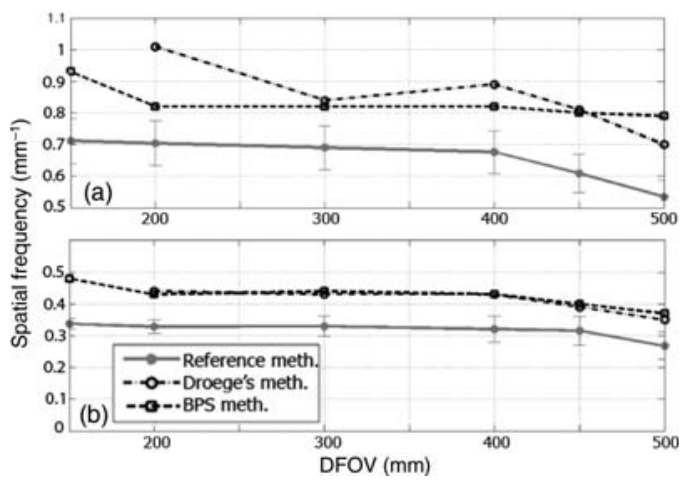

Figure 5. Comparison of spatial frequency results extracted at (a) $5 \%$ and (b) $50 \%$ of the MTFs obtained with the different methods when varying the DFOV. The acquisition was performed with the standard reconstruction filter and a $\mathrm{CTDI}_{\mathrm{vol}}=100 \mathrm{mGy}$.

Effects of the DFOV are presented in Figure 5. Significant fluctuations were observed for Droege's method for DFOV values between 200 and $500 \mathrm{~mm}$ (Figure 5a), whereas an excellent agreement between Droege's and BSP methods was obtained for the same DFOV values (Figure 5b). For larger DFOVs, $f_{\text {nyquist }}$ affected the shape of the MTF and produced an important decrease of spatial frequencies at $5 \%$, while the decrease was less strong at $50 \%$. The same trend was visible for the reference method. Note that for this latter method, incertitude was taken into account. As error bars depend on the DFOV (pixel size), even with a pre-sampled LSF, their size increases when DFOV increases.

\section{DISCUSSION}

\section{QualiFormeD: Routine stability control}

In routine QC procedures, the constancy of the spatial resolution is usually monitored through a stability control. In this control, the MTF spatial frequencies corresponding to $50 \%, 10 \%, 5 \%$ and $2 \%$ of the MTF (Figure 2) are compared with pre-established values and tolerances considered as 'reference' values. The technique adapted to such controls should be able to perform a fast and simple analysis in the range of conditions matching those of the clinical protocol.

\section{Noise effect}

Although the noise can be reduced when the dose is increased, it cannot be totally avoided. With sharp reconstruction kernels, such as the lung, the noise is able to affect the MTF computation and to modify the spatial resolution measurements. Based on the results shown in Figure 4, one can observe that the
BPS method is more sensitive to noise increase than Droege's method. Indeed, the amplitude of the maximum variation produced with the BPS method was around $10 \%$ for a range of $\mathrm{CTDI}_{\mathrm{vol}}$ extended from 100 to $5 \mathrm{mGy}$. Below this value, the bead object is drowned by the noise and even manual detection becomes impossible. On the other hand, the constancy of the results can be attributed to the bar patterns that are visible even for very high noise.

One can conclude that the Droege's method is particularly well-adapted for assessing the spatial frequency in the case of low dose protocols and that the frequency approximation use for a frequency lesser than $f_{\mathrm{c}} / 3$ does not significantly limit the determination of spatial frequency at MTF levels around $50 \%$ and lower.

\section{DFOV effect}

In Figure 5, while an excellent agreement was observed between Droege's and the BPS method for results at $50 \%$ of the MTFs when varying the DFOVs from 200 to $400 \mathrm{~mm}$, significant fluctuations (about $20 \%$ ) were visible for Droege's results at $5 \%$ of the MTFs. The reason for this behaviour is attributed to a slight misalignment of the QualiMagiQ detection system owing to a particularity of GE CT scanners. It has been shown that GE CT images were shifted when the DFOV and the reconstruction kernel were modified.

For a DFOV $=300 \mathrm{~mm}$, on which the setting of the QualiMagiQ detection system was adjusted, a good concordance is achieved with respect to the BPS method (Figure 5b). However, for other DFOV values, the previous effect slightly shifts the automatic detection pattern of the high frequency with respect to the ones of the Catphan 600 phantom bar patterns providing fluctuations of spatial frequencies extracted at $5 \%$.

For smaller DFOV values, the diameter of the Catphan 600 phantom $(200 \mathrm{~mm})$ is larger than the image DFOV. QualiMagiQ automatic detection is based on the phantom edge detection. Thus, images with DFOVs $<200 \mathrm{~mm}$ cannot be analysed with Droege's method.

For a range of DFOV from $500 \mathrm{~mm}$ to $150 \mathrm{~mm}$ and for $\mathrm{CTDI}_{\mathrm{vol}}$ values down to $2.5 \mathrm{mGy}$, QualiFormeD's analysis provided reliable results every time, either with Droege's method or the BSP method. This fast and simple-to-use software is thus quite well-adapted for routine QC.

However, the overestimation of the absolute values between the QualiFormeD's methods and the reference method as well as the finite application domain of QualiMagiQ suggests that this software is not directly adapted for the in-depth controls required in a commissioning programme. 


\section{AUTOMATIC MTF ASSESSMENT METHOD FOR COMMISSIONING PROGRAMME}

\section{COMMISSIONING PROGRAMME}

Previous results have shown that both Droege's and BPS methods have intrinsic limitations. Droege's method is inaccurate for frequencies lower than $f_{\mathrm{c}} / 3$ and can be influenced by the presence of artefacts created using dense material (aluminium bars) as shown in a paper by Torfeh et al. ${ }^{(8)}$. In the same way, the BPS method is based on a small number of meaningful points, making this technique noise-sensitive. In order to make this method reliable, fit functions are usually required to smooth MTF results but these functions strongly affect MTFs and thus the extraction of spatial frequency values.

On the other hand, Boone's method is robust with respect to aliasing, more resilient to noise and provides absolute measurements. In the situation of CT characteristic comparisons, this method may provide a link between relative values (obtained with previous methods) and absolute values, making comparisons between different routine QC programmes and CT units possible. Furthermore, in the context of CT unit commissioning programmes, in which the suitability of the CT unit has to be ensured, while baseline as well as tolerance values have to be established ${ }^{(1)}$, Boone's method can be particularly relevant.

Figure 6 presents two MTFs reconstructed using the soft and bone filters. While a DFOV $=250 \mathrm{~mm}$ does not limit the MTF cut-off frequency obtained with the soft reconstruction filter, the same DFOV value strongly reduces the cut-off frequency of the bone reconstruction. A comparison between the pre-sampled and post-sampled MTFs shows the under-sampling effect produced by this DFOV value. This also means that if the structure size to diagnose is smaller than this cut-off frequency, no detection will be possible. Boone's method, because

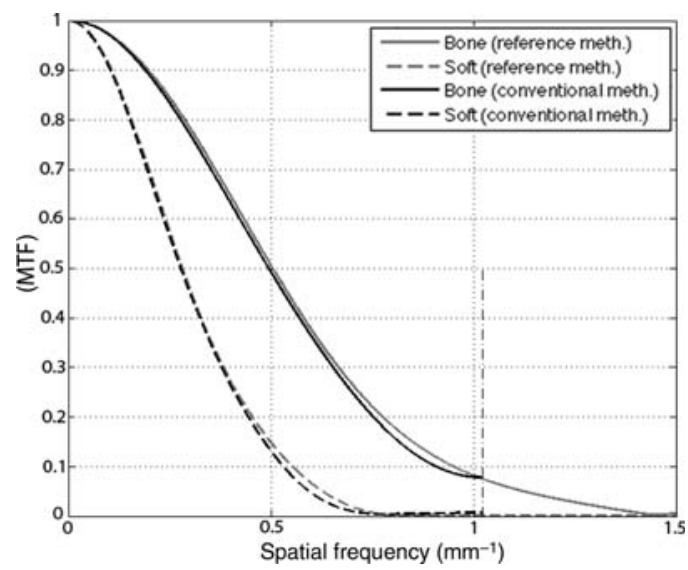

Figure 6. Effect of a fixed DFOV $=250 \mathrm{~mm}$ on MTFs obtained with a bone reconstruction kernel and a soft reconstruction kernel. of its extending range of applications and its ability to provide absolute spatial frequency values, may be an excellent tool to develop clinical protocols using objective criteria.

\section{CONCLUSION}

In this investigation, Boone's ${ }^{(5)}$ pre-sampled MTF technique was used to assess the spatial resolution of a CT scanner. The results were compared with the ones based on Droege's and the BPS methods and carried out with an automatic approach dedicated to routine QC (QualiMagiQ). For Droege's and the BPS methods, results obtained with the automatic approach when varying the DFOV and the CTDI ${ }_{\mathrm{vol}}$ show that this software is well-adapted for stability controls of clinical protocols (typically for DFOV values from $500 \mathrm{~mm}$ to $150 \mathrm{~mm}$ and for CTDI $_{\mathrm{vol}}$ values down to $2.5 \mathrm{mGy}$ ). However, for commissioning programmes, where absolute values are required in extended CT testing conditions, a different method should be used. It was shown that the main benefit of Boone's method is reliability when noise and DFOV increases, because of the larger phantom and the pre-sampled LSF.

In order to use this method in commissioning programmes, a dedicated routine could be added to the present version of the programme investigated in this study, together with the use of a modified phantom. Thus, small-angled horizontal and vertical thin aluminium foils could be added to the Catphan 600 phantom to perform absolute spatial resolution measurements of the different scanning planes of the CT scanners, mainly, transverse, coronal and sagittal planes. Based on these MTF results, a noise equivalent quanta metric could be developed to help estimate low-contrast detection performances.

\section{FUNDING}

This work was partially supported by the Swiss National Science Foundation (no. 3240-120382).

\section{REFERENCES}

1. Aroua, A., Trueb, Ph., Vader, J.-P., Valley, J.-F. and Verdun, F. R. Exposure of the Swiss population by radiodiagnostics: 2003 review. Health Phys. 92, 442-448 (2007).

2. ImPACT. CT scanner acceptance testing. Leaflet No. 1, v. 1.02 (2001).

3. International Commission on Radiological Protection. Managing patient dose in computed tomography. ICRU Publication 87 (Oxford: Pergamon Press) (2000).

4. Droege, R. T. and Morin, R. L. A practical method to measure the MTF of CT scanners. Med. Phys. 9, 758-760 (1982).

5. Boone, J. M. Determination of the presampled MTF in computed tomography. Med. Phys. 28, 356-360 (2001). 


\section{F. MIÉVILLE ET AL.}

6. Judy, P. F. The line spread function and modulation transfer function of a computed tomographic scanner. Med. Phys. 3, 233-236 (1976).

7. Fujita, H., Tsai, D.-Y., Itoh, T., Doi, K., Morishita, J., Ueda, K. and Ohtsuka, A. A simple method for determining the modulation transfer function in digital radiography. IEEE Trans. Med. Imag. 11, 34-39 (1992).

8. Torfeh, T., Beaumont, S., Guedon, J.-P. and Denis, E. Evaluation of two software tools dedicated to an automatic analysis of the CT scanner image spatial resolution. IEEE Eng. Med. Biol. Soc. Lyon, France, August 2007. 